\title{
Response to commentary
}

\author{
Colleen D. McDermott ${ }^{1}$ (1)
}

Received: 30 May 2021 / Accepted: 12 June 2021 / Published online: 8 July 2021

(C) The International Urogynecological Association 2021

We thank Drs. Azarkish and Janghorban for their interest in our recently published article [1]. The main objective of this study was to help clinicians determine the appropriate interval for office-based pessary care, in an attempt to provide a recommendation on best practice. Patients who perform their own pessary care at home were not included, as their management is completely different and would not provide us with any useful information regarding the objective of this study. To all intents and purposes, our study would be considered a "pilot study," demonstrating that a randomized trial design for this particular question is not feasible. It was neither the questionnaire nor the set interval for pessary follow-up (3 months versus 6 months) that contributed to the poor patient recruitment. It was the fact that patients who require office-based pessary care need individualized scheduling based on personal preferences and objective needs to reduce adverse events.

\section{Reference}

1. Anglim B, Zhao ZI, Lovatsis D, McDermott CD. The extended pessary interval for care (EPIC) study: a failed randomized clinical trial. Int Urogynecol J. 2021;32:937-44

Publisher's note Springer Nature remains neutral with regard to jurisdictional claims in published maps and institutional affiliations.
Colleen D. McDermott

colleen.mcdermott@utoronto.ca

1 Division of Female Pelvic Medicine and Reconstructive Pelvic Surgery, Department of Obstetrics and Gynaecology, Mount Sinai Hospital, University of Toronto, 8-815, 700 University Avenue, Toronto, ON, Canada 